\title{
Viral Hepatitis and Its Complications
}

\author{
Stefan Zeuzem ${ }^{a}$ Wolf O. Bechstein ${ }^{b}$ \\ ${ }^{a}$ Medizinische Klinik 1, Universitätsklinikum Frankfurt, Frankfurt am Main, Germany; \\ ${ }^{b}$ Klinik für Allgemein- und Viszeralchirurgie, Universitätsklinikum Frankfurt, Frankfurt am Main, Germany
}

Recent advances in the management of viral hepatitis and its complications have prompted us to ask leading scientists to give their current views on the state-of-the-art management of these often heterogeneous and complicated disease courses.

Manka et al. [1] have summarized the recent knowledge on liver failure due to acute viral hepatitis (A-E), emphasizing that hepatitis $\mathrm{E}$ virus is an emergent disease in Europe and that reactivation of hepatitis $\mathrm{B}$ virus (HBV) under chemotherapy is also an increasing problem. When acute liver failure develops due to HBV infection, the immediate treatment with nucleotide or nucleoside analogs (NUC) is well tolerated and beneficially affects the outcome.

When cirrhosis is established due to HBV infection alone or associated with hepatitis D virus (HDV) infection, the treatment options are both pegylated interferon and NUC. Höner zu Siederdissen and Cornberg [2] discuss the current knowledge on the management of cirrhosis due to HBV with or without HDV co-infection.

There has been tremendous progress in the field of management of hepatitis $\mathrm{C}$ virus (HCV)-associated liver cirrhosis. Various antiviral treatment approaches with NS3/4A inhibitors, NS5A inhibitors as well as nucleoside and non-nucleoside NS5B inhibitors are elegantly discussed by Schneider and Sarrazin [3].

For many patients, liver transplantation may be indicated in complicated cirrhosis. In case of viral origin of the cirrhosis, prevention of re-infection of the graft remains one of the priorities to ensure long-term success. Antiviral treatment can be initiated before or after liver transplantation, with the various strategies regarding pre- and post-transplant antiviral therapy for HBV and $\mathrm{HCV}$ infection being outlined by Welker and Zeuzem [4].
In a patient with established cirrhosis, this in itself is a precancerous condition calling for surveillance strategies to detect hepatocellular carcinoma (HCC) at an early, potentially curable stage.

Marquardt et al. [5] give an excellent and up-to-date review on screening strategies and detection algorithms for patients who are at risk of developing HCC and emphasize that these algorithms should be strictly applied.

When HCC is diagnosed, today we have a wide armamentarium of therapeutic strategies including liver resection and transplantation, local ablation techniques, transarterial chemoembolization, and medical therapy with sorafenib. The standards of current treatment together with an outlook on future developments are delineated by Trojan et al. [6].

Furthermore, antiviral treatments may play a role both in the prevention of HCC as well as during the treatment of HCC. The different strategies in this particular field are outlined by Roderburg et al. [7].

Finally, an expert panel of experienced clinicians in this field comprising Prof. Galle, Prof. Nashan, Prof. Pratschke, Prof. Ricke, and Prof. Vogl provide insights into the interdisciplinary treatment and management of HCC in the context of viral hepatitis [8].

While progress has certainly been made, there still remains much to be done. By implementing the current knowledge into clinical practice lives may be saved, and we hope that the readers may find the information presented in this special issue helpful for their daily clinical work.

\section{References}

1 Manka P, Verheyen J, Gerken G, Canbay A: Liver failure due to acute viral hepatitis (A-E). Visc Med 2016; 32:DOI: 10.1159/000444915.

2 Höner zu Siederdissen C, Cornberg M: Management of $\mathrm{HBV}$ and $\mathrm{HBV} / \mathrm{HDV}$-associated liver cirrhosis. Visc Med 2016;32:DOI: 10.1159/000445518.

3 Schneider MD, Sarrazin C: Management of HCV-associated liver cirrhosis. Visc Med 2016;32:DOI: 10.1159/ 000445330 .
4 Welker M-W, Zeuzem S: Pre- and post-transplant antiviral therapy (HBV, HCV). Visc Med 2016;32:DOI: $10.1159 / 000445152$.

5 Marquardt JU, Nguyen-Tat M, Galle PR, Wörns MA: Surveillance of hepatocellular carcinoma and diagnostic algorithms in patients with liver cirrhosis. Visc Med 2016;32:DOI: 10.1159/000445407.

6 Trojan J, Zangos S, Schnitzbauer AA: Diagnostics and treatment of hepatocellular carcinoma in 2016: standards and developments. Visc Med 2016;32:DOI: $10.1159 / 000445730$.
7 Roderburg C, Tacke F, Trautwein C: Antiviral therapy in patients with viral hepatitis and hepatocellular carcinoma: indications and prognosis. Visc Med 2016;32: DOI: $10.1159 / 000444990$.

8 Bechstein WO (Chair): Viral hepatitis and its complications: management of hepatocellular carcinoma (Interdisciplinary Discussion). Visc Med 2016;32:DOI: $10.1159 / 000445306$.

\section{KARGER \\ Fax +497614520714

